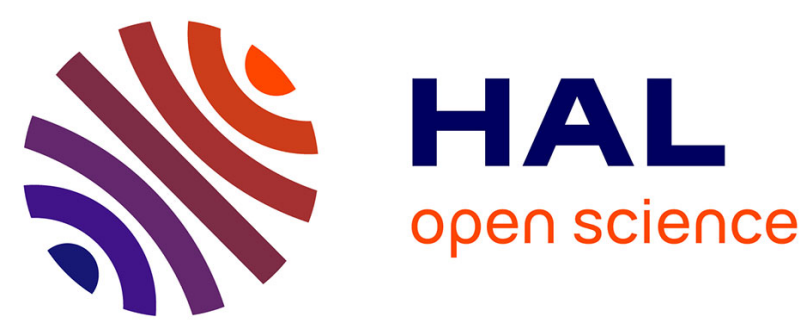

\title{
Vacancy and interstitial defects in the vortex lattice of superconducting thin films
}

\author{
Enrick Olive, Ernst Helmut Brandt
}

\section{To cite this version:}

Enrick Olive, Ernst Helmut Brandt. Vacancy and interstitial defects in the vortex lattice of superconducting thin films. Physical Review B: Condensed Matter and Materials Physics (1998-2015), 1999, 59 (10), pp.7116-7122. 10.1103/PhysRevB.59.7116 . hal-01794614

\section{HAL Id: hal-01794614 \\ https://hal.science/hal-01794614}

Submitted on 17 May 2018

HAL is a multi-disciplinary open access archive for the deposit and dissemination of scientific research documents, whether they are published or not. The documents may come from teaching and research institutions in France or abroad, or from public or private research centers.
L'archive ouverte pluridisciplinaire HAL, est destinée au dépôt et à la diffusion de documents scientifiques de niveau recherche, publiés ou non, émanant des établissements d'enseignement et de recherche français ou étrangers, des laboratoires publics ou privés. 


\title{
Vacancy and interstitial defects in the vortex lattice of superconducting thin films
}

\author{
Enrick Olive* and Ernst Helmut Brandt \\ Max Planck Institut für Metallforschung, D-70506 Stuttgart, Germany
}

(Received 21 July 1998)

\begin{abstract}
A method developed previously for the lattice of infinitely long parallel Abrikosov vortices in type-II superconductors is extended to vortices in thin superconducting films. The self-energy and interaction energy of vacancies and interstitials in the triangular lattice of vortices in the film are calculated within London theory. Various stable and metastable equilibrium configurations of the flux-line lattice around such point defects are found, similar to the result for long vortex lines. In particular, the vacancy with highest (sixfold) symmetry usually does not exhibit the lowest energy, and the defect energies are very small compared with the binding energy of one vortex due to the relaxation of the surrounding vortex lattice. The interaction between point defects is weak, but is attractive as opposed to the long parallel Abrikosov vortex case where the defect interaction can be repulsive in some cases. [S0163-1829(99)00410-5]
\end{abstract}

\section{INTRODUCTION}

In a recent paper the energy and local structure of vacancies and interstitials were calculated for the triangular lattice of parallel Abrikosov vortices (flux lines) in bulk type-II superconductors, ${ }^{1}$ continuing previous numerical, ${ }^{2,3}$ and analytical $^{2-5}$ calculations. In the present paper, the energy and local symmetry of such point defects are calculated for the lattice of short vortices occurring in thin superconductor films in an applied magnetic field. As in Ref. 1, we consider the London limit of nonoverlapping vortex cores; this is the region where the Ginzburg-Landau order parameter goes to zero over a distance $\xi$, the Ginzburg-Landau coherence length. In the London approximation, the spacing of all vortices should be much larger than the core radius. This condition typically is satisfied when the average magnetic induction $B=2 \Phi_{0} / \sqrt{3} a^{2}$ is much smaller than the upper critical field $B_{c 2}=\Phi_{0} /\left(2 \pi \xi^{2}\right)$. Here $\Phi_{0}=h / 2 e=2 \times 10^{-15} \mathrm{Tm}^{2}$ is the quantum of magnetic flux and $a=\left(2 \Phi_{0} / \sqrt{3} B\right)^{1 / 2}$ the spacing of the ideal triangular vortex lattice.

In the London limit the energy of any arrangement of parallel (long or short) vortices in isotropic or uniaxial superconductors is the sum over all pair interactions $V\left(r_{i j}\right)$ where $r_{i j}=\left|\mathbf{r}_{i}-\mathbf{r}_{j}\right|$ is the distance of two vortices with positions $\mathbf{r}_{i}$ and $\mathbf{r}_{j}$. If the vortices are along $z$ one has $\mathbf{r}_{i}$ $=\left(x_{i}, y_{i}\right)$. The rotationally symmetric pair potential $V\left(r_{i j}\right)$ has a rotationally symmetric Fourier transform $\tilde{V}(k)$,

$$
V(r)=\int \frac{d^{2} k}{4 \pi^{2}} \tilde{V}(k) e^{i \mathbf{k r}} .
$$

For long vortex lines of length $L$ the London potential is

$$
\tilde{V}(k)=E_{0} \frac{2 \pi L}{k^{2}+\lambda^{-2}}
$$

with prefactor $E_{0}=\Phi_{0}^{2} /\left(2 \pi \mu_{0} \lambda^{2}\right)$ (energy per unit length) and range $\lambda$, the magnetic penetration depth. Equations (1) and (2) yield $V\left(r_{i j}\right)=E_{0} K_{0}\left(r_{i j} / \lambda\right)$ where $K_{0}(x)$ is a modified Bessel function having the limits $K_{0}(x) \approx \ln (1 / x) \quad(x$ $\ll 1)$ and $K_{0}(x) \approx(\pi / 2 x)^{1 / 2} \exp (-x) \quad(x \gg 1)$. For thin films with thickness $d \ll \lambda$ in a perpendicular field, the interaction potential of the short vortices with length $d$ is 6,7

$$
\tilde{V}(k)=E_{0} \frac{2 \pi d}{k^{2}+k \Lambda^{-1}} .
$$

The effective range of this potential is

$$
\Lambda=\frac{2 \lambda^{2}}{d} \gg \lambda \text {. }
$$

The interaction (3) applies not only to thin films but (with slight modifications) also to the pancake vortices in one of the superconducting $\mathrm{CuO}$ layers in high-temperature superconductors. ${ }^{8-11}$

In bulk superconductors the London approximation is valid if $B \ll B_{c 2}$ and if $\lambda>1.4 \xi$, i.e., if the Ginzburg-Landau parameter is $\kappa=\lambda / \xi>1.4$. This is so since, in addition to the magnetic repulsion of range $\lambda$, long vortex lines exhibit another interaction which originates from the overlap of the vortex cores and which has a term with the range $\sqrt{2} \xi$. $^{12-14}$ At large distances $r_{i j} \gg \lambda$ this term dominates when $\kappa$ $<1.4$; in this case the London approximation does not hold. Therefore, a further condition for the London picture to apply is usually written as $\kappa \gg 1$. However, for thin films this condition is not required since the long-range magnetic repulsion always dominates because of $\Lambda \gg \lambda$.

Note the similarity of the two potentials (2) and (3). In particular, for large $k$ values, corresponding to short distances, both Fourier transforms decrease as $1 / k^{2}$, therefore, both potentials at short distances $\left(r_{i j} \ll \lambda\right.$ or $\left.r_{i j} \ll \Lambda\right)$ coincide and take the form

$$
V\left(r_{i j}\right)=E_{0} \ln \left(\frac{\text { const }}{r_{i j}}\right) \times \text { vortex length. }
$$

At large distances the interaction between long vortices decreases exponentially, $V\left(r_{i j}\right) \propto \exp \left(-r_{i j} / \lambda\right)$, but the interaction between vortices in thin films decreases very slowly,

$$
V\left(r_{i j}\right) \propto 1 / r_{i j} \text { for } r_{i j} \gg \Lambda \text {. }
$$


This long-range interaction is due to the magnetic stray field generated by the ends of the vortices, which behave like magnetic monopoles. For the interaction of vortices in thicker films see Refs. 15,16.

\section{EFFECTIVE INTERACTION POTENTIAL}

As in Ref. 1 we use periodic boundary conditions in our computation. The interaction energy $U_{N}$ of the $N$ vortices per super cell may thus be written in the form

$$
U_{N}=\frac{1}{2} \sum_{i=1}^{N} \sum_{j=1}^{\infty}{ }^{\prime} V\left(r_{i j}\right)=\frac{1}{2} \sum_{i=1}^{N} \sum_{j=1}^{N}{ }^{\prime} V_{e}\left(\mathbf{r}_{i j}\right)+\frac{N}{2} U_{\mathrm{per}},
$$

where the dash at the $j$ sum excludes the term $j=i . V_{e}(\mathbf{r})$ is an effective interaction potential which includes the interaction with the images in all other cells, i.e., at the positions $\mathbf{r}_{i}+\mathbf{R}$ where $\mathbf{R}=\mathbf{R}_{m n}=\left(m L_{x}, n L_{y}\right)(m, n$ integer $)$ are the lattice vectors of the rectangular periodicity cells. For the longvortex interaction, Eq. (2), this effective potential is given by Eq. (5) of Ref. 1. For thin films with the potential (3), the infinite sums in Eq. (7), however, diverge for an infinitely large system (infinite number of super cells) due to the long range of $V\left(r_{i j}\right) \propto 1 / r_{i j}$. One, therefore, has to subtract a constant which removes the divergence. This subtraction of a constant does not alter the difference of the energies of different vortex arrangements with same number of vortices per super cell, which we intend to compute. A natural choice of this constant is the (also diverging) average of $V\left(r_{i j}\right)$, i.e., we replace $V$ by $V-\langle V\rangle$. This results in an effective potential of the form

$$
\begin{aligned}
V_{e}(\mathbf{r}) & =\sum_{\mathbf{R}}(V(\mathbf{r}+\mathbf{R})-\langle V(\mathbf{r})\rangle) \\
& =\frac{1}{A} \sum_{\mathbf{K} \neq \mathbf{0}} \tilde{V}(K) \cos \mathbf{K r}=\frac{2 \pi}{A} \sum_{\mathbf{K} \neq \mathbf{0}} \frac{\cos \mathbf{K} \mathbf{r}}{K^{2}+K \Lambda^{-1}}
\end{aligned}
$$

in energy units $E_{0} d$. Here $\mathbf{K}=\mathbf{K}_{\mu \nu}=2 \pi\left(\mu / L_{x}, \nu / L_{y}\right)$ are the reciprocal-lattice vectors of the superlattice with the rectangular basic cell of area $A=L_{x} L_{y}$. The subtraction of the spatial average $\left\langle V\left(r_{i j}\right)\right\rangle$ has modified the $\mathbf{K}$ sums such that the term $\mathbf{K}=0$ vanishes.

As in Ref. 1, the correction constant $N U_{\text {per }} / 2$ in Eq. (7) originates from the interaction of each vortex with its own images, which had dropped out from the sum over $V_{e}$ in Eq. (7) when the terms $j=i$ were omitted. This term does not depend on the vortex positions, like the vortex self-energies, which are omitted in the interaction (7). Since $N U_{\text {per }}$ depends on the periodicity lengths $L_{x}$ and $L_{y}$, this term is important when the energies of periodicity cells of various shape or size are compared. In particular, if $U_{\text {per }}$ were disregarded, the interaction energy per vortex $U_{N} / N$ of the infinitely large ideal flux-line lattice (FLL) artificially would depend on the choice of the periodicity area. Explicitly one has

$$
\begin{aligned}
U_{\mathrm{per}} & =\sum_{\mathbf{R} \neq 0}(V(R)-\langle V(r)\rangle) \\
& \approx \frac{1}{A} \sum_{\mathbf{K} \neq \mathbf{0}} \tilde{V}(K)-\int_{k>k_{\mathrm{BZ}}} \frac{d^{2} k}{4 \pi^{2}} \tilde{V}(k) \\
& \approx \frac{2 \pi}{A} \sum_{K \leqslant k_{1}} \frac{1}{K^{2}+K \Lambda^{-1}}-\ln \frac{1+k_{1} \Lambda}{1+k_{\mathrm{BZ}} \Lambda} .
\end{aligned}
$$

Here the $k$ integral is over the area outside the first Brillouin zone, which may be approximated by a circle of radius $k_{\mathrm{BZ}}$ $=(4 \pi / A)^{1 / 2}$, and $k_{1} \gg k_{\mathrm{BZ}}$ is an arbitrary cutoff radius to be chosen sufficiently large such that the sum contains many terms. In this last sum the dash means omission of the term $\mathbf{K}=0$. The inaccuracies in Eq. (9) correspond to neglecting a constant contribution, independent of the size and shape of the supercell. This constant may be absorbed in the vortex self-energy, which is disregarded in our calculations since it drops out from all our final results.

In a similar way, the interaction energy of ideal periodic vortex lattices may be computed as $U_{N}=N U_{B}$, where $U_{B}$ is the binding energy of one vortex defined by

$$
\begin{aligned}
U_{\mathrm{B}}\left(\frac{a}{\Lambda}\right) & =\frac{1}{2} \sum_{\mathbf{R} \neq 0}(V(R)-\langle V(r)\rangle) \\
& \approx \frac{\pi B}{\Phi_{0}} \sum_{K \leqslant k_{1}} \frac{1}{K^{2}+K \Lambda^{-1}}-\frac{1}{2} \ln \frac{1+k_{1} \Lambda}{1+k_{\mathrm{BZ}} \Lambda},
\end{aligned}
$$

where now the $\mathbf{R}$ and $\mathbf{K}$ are interpreted as the vectors and reciprocal vectors of the triangular vortex lattice, which has a unit cell area $\Phi_{0} / B=a^{2} \sqrt{3} / 2$, where $a$ is the spacing of the ideal FLL, and $K^{2}=K_{m n}^{2}=\left(16 \pi^{2} / 3 a^{2}\right)\left(m^{2}+m n+n^{2}\right)(m, n$ integer). The radius of the circularized Brillouin zone is now $k_{\mathrm{BZ}}=\left(4 \pi B / \Phi_{0}\right)^{1 / 2}$, and the cutoff radius is $k_{1} \gg k_{\mathrm{BZ}}$.

As discussed in Ref. 1, for evaluating the $\mathbf{K}$ sums it is convenient to multiply $\tilde{V}(k)$ by a cutoff factor $f(k)$ which decreases as $\exp \left(-r_{0}^{2} k^{2} / 4\right)$ for large $k, V(k) \rightarrow V(k) f(k)$. Here we shall choose this factor as

$$
f(k)=\exp \left(-\frac{r_{0}^{2} k^{2}}{4}\right)\left(1+\frac{r_{0}^{2} k^{2}}{4}\right)
$$

with a small radius $r_{0}$. We choose $r_{0}=a / 20$ where $a$ is the average vortex spacing. ${ }^{1}$ The cutoff factor (11) has zero curvature at $k=0,\left.\nabla^{2} f(k)\right|_{k=0}=0$. It thus practically does not modify the potential $V\left(r_{i j}\right)$ at large distances $r_{i j} \gg r_{0}$, but at small $r$ it means a convolution of $V\left(r_{i j}\right)$ with a Gaussian $\left(1 / \pi r_{0}^{2}\right) \exp \left(-r^{2} / r_{0}^{2}\right)$ and multiplication with the operator (1 $\left.-r_{0}^{2} \nabla^{2} / 4\right)$. [In Ref. 1 we divided the convoluted $V\left(r_{i j}\right)$ by $1+r_{0}^{2} \nabla^{2} / 4=1-r_{0}^{2} / 4 \lambda^{2}$, which had approximately the same effect.] This slightly modified potential turns out to give very accurate numerical results comparable to those obtained by an Ewald sum technique. We thus insert $V(k) f(k)$ in Eqs. $(8)$ and (9) which we use for our computations.

The energy of a superlattice of defects is defined as described in Sec. II C of Ref. 1. For point defects with $n$ vortices added to the supercell (e.g., $n=-2,-1,1,2$, respectively, for double vacancy, vacancy, interstitial, and double interstitial) this defect energy per supercell is 


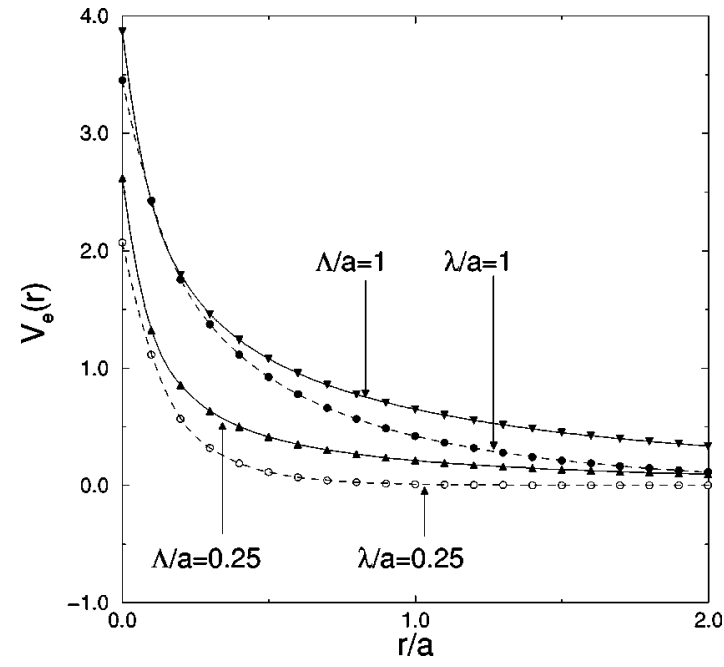

FIG. 1. Effective interaction potential between two vortices $i$ and $j$ with distance $r$ inside the periodicity cell. This potential includes the interaction with all "images" translated by vectors $\mathbf{R}_{m n}=\left(m L_{x}, n L_{y}\right)(m, n$ integer $)$. Shown is the effective potential at small distances for a much larger periodicity cell of size $\left(L_{x}, L_{y}\right)$ $=(25,30 \sqrt{3} / 2) a$. Shown are the effective potential for thin films (solid line), see Eq. 8, and the effective potential for parallel Abrikosov vortices in bulk type-II superconductors (dashed line), see Eq. (5) in Ref. 1. We display these effective potentials for different values of the effective penetration depth $\Lambda / a=1$ and 0.25 for thin films, and for $\lambda / a=1$ and 0.25 for long vortices.

$$
\begin{aligned}
U_{\mathrm{def}}= & U_{N+n}^{\mathrm{def}}-U_{N+n}^{\mathrm{ideal}} \\
= & U_{N+n}^{\mathrm{def}}-\frac{N+2 n}{N} U_{N}^{\text {ideal }}+(N+2 n) U_{B}\left(a_{N}\right) \\
& -(N+n) U_{B}\left(a_{N+n}\right),
\end{aligned}
$$

where $U_{B}(a)$ is the binding energy of one vortex as given by Eq. (10).

The lattice relaxation was performed by the same quasistatic method described in Ref. 1. We again consider periodically arranged point defects with periodicity cells of various sizes $\left(L_{x}, L_{y}\right)=(5,6 \sqrt{3} / 2) t a$, where $t=1 \ldots 8$ is an integer and $a$ the lattice spacing. In this way, the rectangular cell approximates a square to within $4 \%$. For $t=8$, the periodicity cell contains $40 \times 48=1920$ vortices.

As mentioned in the Introduction, the interaction potential for long or short vortices is logarithmic if $\lambda \gg r_{i j}$ or $\Lambda$ $\gg r_{i j}$, respectively. For $\Lambda / a \gg 1$, we thus expect similar results as those presented in Ref. 1 for long vortices with $\lambda / a \gg 1$. Therefore, we only computed small values of $\Lambda / a$, namely, $\Lambda / a=1$ and $\Lambda / a=0.25$. Figure 1 shows the effective potential of long vortices for $\lambda / a=1$ and $\lambda / a=0.25$, compared with the potential of short vortices for $\Lambda / a=1$ and $\Lambda / a=0.25$.

\section{RESULTS}

\section{A. Vacancies}

To create a superlattice of vacancies we start from the ideal triangular lattice and remove one flux line per supercell. After relaxation we then still find three different equilibrium configurations exhibiting six-, three-, and twofold
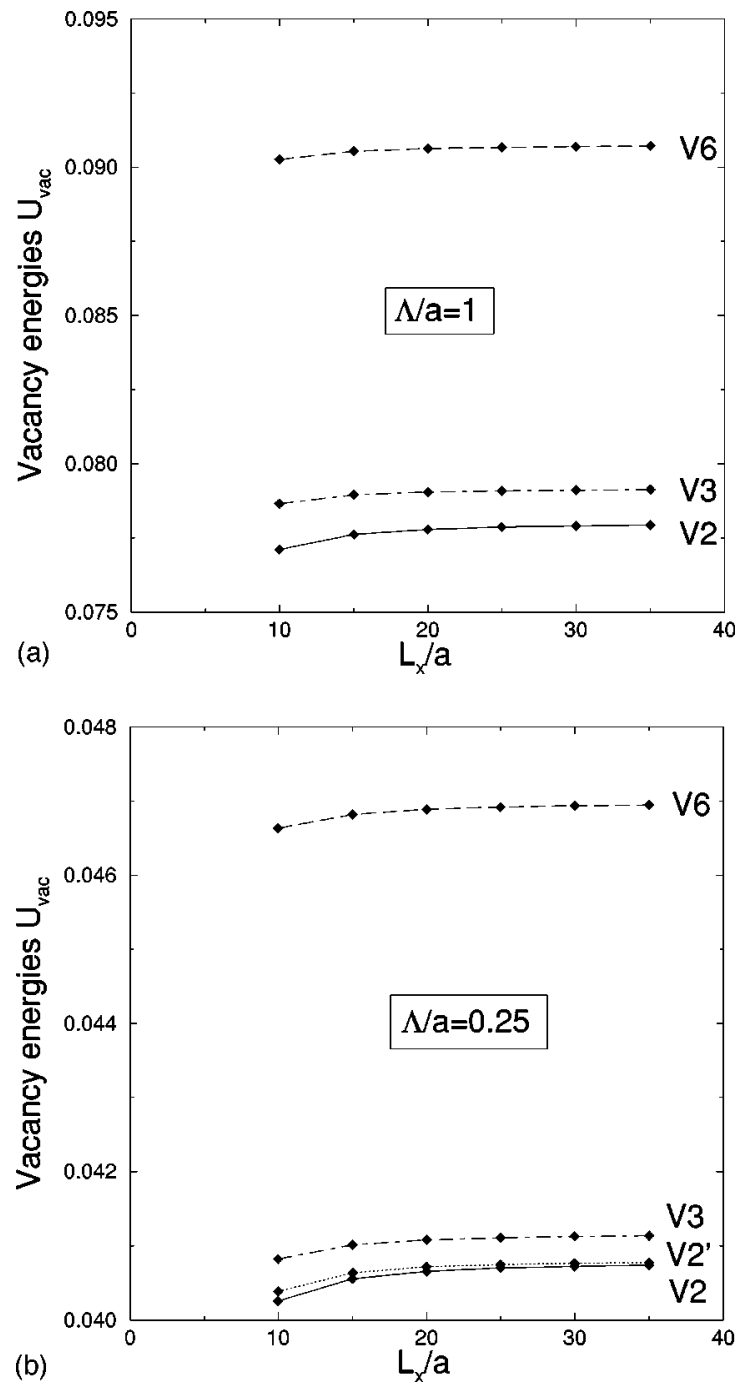

FIG. 2. Supercell-size (or distance) dependence of the defect energies for the sixfold symmetric vacancy V6 (dashed line), threefold symmetric vacancy V3 (dash-dotted line), and twofold symmetric vacancy V2 (solid line). The energy in units of $E_{0}$ is plotted versus $L_{x} / a$, where $L_{x}$ and $L_{y}=1.04 L_{x}$ are the sides of the rectangular periodicity box and $a$ is the lattice spacing. Results are presented for (a) $\Lambda / a=1$ and (b) $\Lambda / a=0.25$. The lower the symmetry is, the lower is the vacancy energy. For both values of $\Lambda / a$, the configuration V2' (twofold symmetric configuration where the symmetry axis goes through a vortex position) has a higher energy than the V2 configuration (twofold symmetric configuration where the symmetry axis goes through the initial vacancy position). Note that with long vortices, for $\lambda / a=0.25$ the V2' configuration had a lower energy than the V2 configuration (see Ref. 1). Furthermore, increasing energies with increasing $L_{x} / a$ show that the interaction between vacancies (whatever their symmetry) is attractive for distances $L_{x}$ $>10 a$.

axial symmetry around the vacancy (V6, V3, V2), see Fig. 1 in Ref. 1 for such final configurations. These configurations are metastable states since our "viscous" relaxation method remains stationary at these configurations during many iteration steps before it continues to find a configuration with lower energy.

The vacancy energies were computed from Eq. (12) with $n=-1$. Our results are displayed in Fig. 2 and Table I. 
TABLE I. Energies of point defects at constant line density $B / \Phi_{0}$ for several sizes of the periodicity cell $\left(L_{x}, L_{y}\right)$ $=(5,6 \sqrt{3} / 2) t a$ with $t=2,3,4,5,6,7$. Listed are the energies of vacancies $\left(U_{\text {vac }}\right)$ and interstitials $\left(U_{\text {int }}\right)$ for various symmetries, see text, for two ranges of the vortex interaction, $\Lambda / a=1$ and 0.25 .

\begin{tabular}{cccccc}
\hline \hline & \multicolumn{5}{c}{$\Lambda / a=1$} \\
$t$ & $U_{\text {vac }}^{\mathrm{V} 6}$ & $U_{\text {vac }}^{\mathrm{V} 3}$ & $U_{\text {vac }}^{\mathrm{V} 2}$ & $U_{\text {int }}^{\mathrm{EI}}$ & $U_{\text {int }}^{\mathrm{CI}}$ \\
\hline
\end{tabular}

$\begin{array}{lllllll}2 & 0.090251 & 0.078644 & 0.077095 & 0.054498 & 0.053667\end{array}$

$\begin{array}{lllllll}3 & 0.090524 & 0.078941 & 0.077607 & 0.054712 & 0.053795\end{array}$

$\begin{array}{lllllll}4 & 0.090620 & 0.079037 & 0.077785 & 0.054783 & 0.053857\end{array}$

$\begin{array}{lllllll}5 & 0.090665 & 0.079081 & 0.077863 & 0.054815 & 0.053887\end{array}$

$\begin{array}{llllll}6 & 0.090689 & 0.079104 & 0.077903 & 0.054832 & 0.053904\end{array}$

$\begin{array}{lllllll}7 & 0.090705 & 0.079119 & 0.077926 & 0.054843 & 0.053915\end{array}$

\begin{tabular}{lcccccc}
\multicolumn{7}{c}{$\Lambda / a=0.25$} \\
$t$ & $U_{\text {vac }}^{\mathrm{V} 6}$ & $U_{\text {vac }}^{\mathrm{V} 3}$ & $U_{\text {vac }}^{\mathrm{V} 2}$ & $U_{\text {vac }}^{\mathrm{V}{ }^{\prime}}$ & $U_{\text {int }}^{\mathrm{EI}}$ & $U_{\text {int }}^{\mathrm{CI}}$ \\
\hline 2 & 0.046631 & 0.040819 & 0.040252 & 0.040382 & 0.028890 & 0.028349 \\
3 & 0.046818 & 0.041014 & 0.040552 & 0.040639 & 0.028981 & 0.028396 \\
4 & 0.046887 & 0.041078 & 0.040655 & 0.040717 & 0.029010 & 0.028421 \\
5 & 0.046919 & 0.041108 & 0.040700 & 0.040749 & 0.029023 & 0.028433 \\
6 & 0.046934 & 0.041124 & 0.040723 & 0.040764 & 0.029030 & 0.028440 \\
7 & 0.046945 & 0.041134 & 0.040735 & 0.040773 & 0.029035 & 0.028444 \\
\hline
\end{tabular}

First, we find that for both ratios of $\Lambda / a$, the lower the symmetry, the lower the final vacancy energy. Note that the twofold symmetry V2' (where the twofold symmetry axis goes through a vortex position, see Fig. 3 in Ref. 1) is still a metastable state in the thin-film case, but for $\Lambda / a=0.25$ it has no longer a lower energy than the usual V2 configuration (where the twofold symmetry axis goes through the initial vacancy position).

Second, since we change only the size of the periodicity cell and not its shape, keeping $L_{x} / L_{y}=0.96$, the total energy of such a superlattice of defects should exhibit qualitatively the same distance dependence as the interaction between two single defects in a large periodicity cell. Thus, increasing energies with increasing size $L_{x} \times L_{y}$ of the periodicity cell means increasing the interaction energy between two single defects with increasing their distance. We therefore conclude that the interaction between two vacancies is attractive for distances between 10 and 35 lattice spacings. This result is valid whatever the vacancy symmetry is.

\section{B. Interstitials}

Starting from the ideal triangular lattice, we added one flux line per supercell to create a superlattice of interstitials. Two different types of interstitials were investigated: the centered interstitial (CI) positioned in the center of a triangle formed by three neighboring flux lines, and the edge interstitial (EI) which sits in the middle between two flux lines. After relaxation both types of interstitials keep their original symmetry, i.e., threefold symmetry for the centered interstitial, and twofold symmetry for the edge interstitial (see Fig. 4 in Ref. 1 for examples of such configurations).

The interstitial energies were computed from Eq. (12) with $n=+1$. Our results are displayed in Fig. 3 and Table I. First, we find that for both ratios $\Lambda / a=1$ and $\Lambda / a=0.25$, the
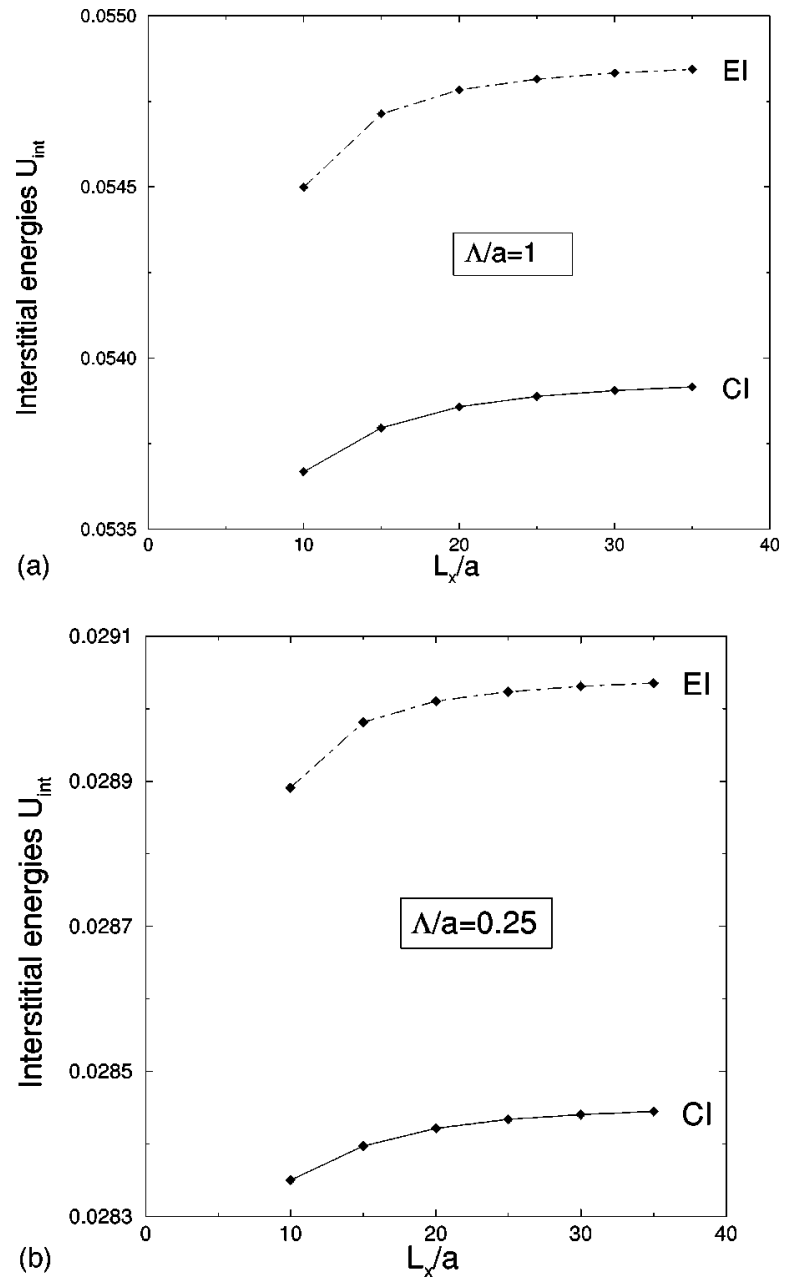

FIG. 3. Supercell size dependence of the defect energies for the centered interstitial (solid line) and for the edge interstitial (dashdotted line). The energy in units of $E_{0}$ is plotted versus $L_{x} / a$ where $L_{x}$ is one side of the (almost quadratic) periodicity box and $a$ is the lattice spacing. Results are presented for (a) $\Lambda / a=1$ and (b) $\Lambda / a$ $=0.25$. For both $\Lambda / a$ values the centered interstitial has a lower energy than the edge interstitial. Thus the higher symmetry now has the lower energy. Furthermore, increasing energies with increasing $L_{x} / a$ show that the interaction between centered or edge interstitials is attractive for distances larger than ten lattice spacings.

CI always has a lower energy than the EI. Thus, in contrast to the vacancies, the higher the symmetry of the interstitial, the lower the final defect energy.

Second, we find increasing energies with increasing size $L_{x} \times L_{y}$ of the periodicity cell, which means increasing interaction energy between two single interstitials with increasing their distance. We therefore conclude that the interaction between two interstitials is attractive for distances between 10 and 35 lattice spacings. This result is valid for both edge (EI) and centered (CI) interstitials. Nevertheless, we obviously expect a repulsive interaction at shorter distances, which should prevent that doubly quantized vortices form. Lastly, note that for $\lambda / a=0.25$ in the long vortex case, ${ }^{1}$ the interaction between CI or EI was repulsive at large distances, as opposed to the attractive interaction we find here for thin films.

Finally, the self-energies of the interstitials are lower than those of the vacancies. Hence, like in the case of long 


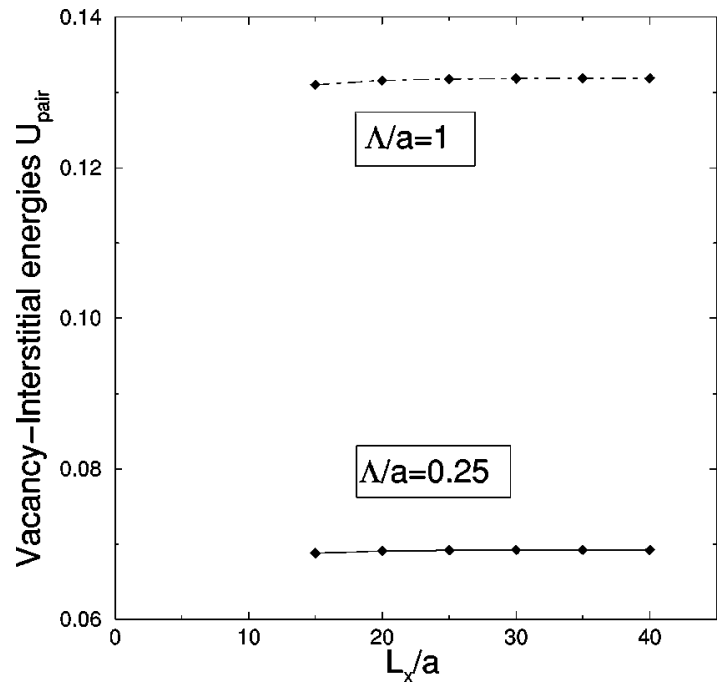

FIG. 4. Supercell size dependence of the energies of the superlattice formed by vacancy-interstitial pairs, for $\Lambda / a=1$ (dash-dotted line) and $\Lambda / a=0.25$ (solid line). The energies in units of $E_{0}$ are plotted versus $L_{x} / a$, where $L_{x}$ is one side of the (almost quadratic) periodicity box and $a$ the lattice spacing. The increasing energies in the range $15 \leqslant L_{x} / a \leqslant 40$ show that the interaction between a vacancy and an interstitial is attractive for distances $s \approx L_{x} / \sqrt{2}$ between $10.6 a$ and $28.3 a$. Furthermore, the interaction between a twofold symmetric vacancy V2 and a threefold symmetric centered interstitial CI results in a symmetry change of the latter when their distance is decreased. Indeed, the interstitial switches to a twofold symmetric interstitial CI' [see Fig. 5(b)] when $L_{x} / a=25$ for $\Lambda / a$ $=1$ and when $L_{x} / a=20$ for $\Lambda / a=0.25$.

vortices, ${ }^{1}$ the interstitials, rather than the vacancies, are energetically favored, and among them the centered interstitial has the lower energy.

\section{Vacancy-interstitial pairs}

We finally consider the case of vortex configurations with one vacancy and one interstitial per periodicity cell, for $\Lambda / a=1$ and $\Lambda / a=0.25$. The vacancy, which we choose as the origin, is created in one corner of the periodicity cell and the interstitial is created as close as possible to the center of the cell. Thus, our periodic boundary conditions yield two interlaced superlattices of vacancies and interstitials. For each size of the periodicity cell $\left(L_{x}, L_{y}\right)$, the closest distance between a vacancy and an interstitial is then $s \approx L_{x} / \sqrt{2}$ and the vector connecting them forms an angle $\alpha \approx \pi / 4$ with the $x$ direction of our periodicity cell, which coincides with one of the three nearest-neighbor directions of the ideal triangular lattice.

The defect energy was computed from Eq. (12) with $n$ $=0$. Our results are displayed in Fig. 4 and Table II. For both values of $\Lambda / a$, we have mentioned that the lower energy configuration for an isolated vacancy corresponds to a twofold symmetry V2, and for an isolated interstitial to a threefold symmetry CI. Thus, when the distance between the two defects of the pair is infinite, we expect the configuration with lower energy to consist of a twofold symmetric vacancy V2 and a centered interstitial CI. This is indeed observed for distances $s \approx L_{x} / \sqrt{2}$ down to $s \approx 17.7 a$ for $\Lambda / a=0.25$, i.e., down to the periodicity cell size $\left(L_{x}, L_{y}\right)=(5,6 \sqrt{3} / 2) 5 a$ [see
TABLE II. Energy of vacancy-interstitial pairs $U_{\text {pair }}$ at constant line density. The periodicity cell sizes are $\left(L_{x}, L_{y}\right)=(5,6 \sqrt{3} / 2) t a$ with $t=3,4,5,6,7,8$. Each cell contains one vacancy and one interstitial at distances $s \approx L_{x} / \sqrt{2}$ The symmetry of each point defect in the pair is also listed.

\begin{tabular}{cccc}
\hline \hline & & $\Lambda / a=1$ & \\
$t$ & $s / a$ & $U_{\text {pair }}$ & Defect symmetries \\
\hline 3 & 10.6 & 0.131037 & V2 and CI' \\
4 & 14.1 & 0.131594 & V2 and CI' \\
5 & 17.7 & 0.131803 & V2 and CI' \\
6 & 21.2 & 0.131860 & V2 and CI \\
7 & 24.8 & 0.131883 & V2 and CI \\
8 & 28.3 & 0.131899 & V2 and CI \\
& & & \\
& & $\Lambda / a=0.25$ & \\
3 & 10.6 & 0.068785 & V2 and CI' \\
4 & 14.1 & 0.069070 & V2 and CI' \\
5 & 17.7 & 0.069171 & V2 and CI \\
6 & 21.2 & 0.069196 & V2 and CI \\
7 & 24.8 & 0.069206 & V2 and CI \\
8 & 28.3 & 0.069212 & V2 and CI \\
\hline \hline
\end{tabular}

the configuration shown in Fig. 5(a) for $s \approx 21.2 a$ ]. But when $s$ is decreased further, qualitative changes are observed. At $s \approx 14.1 a$, i.e., for $\left(L_{x}, L_{y}\right)=(5,6 \sqrt{3} / 2) 4 a$, the vacancy V2 remains unchanged but the symmetry of the interstitial switches from threefold to twofold [see the configuration shown Fig. 5(b) for $s \approx 10.6 a$ ]. The center of symmetry now is no longer at the interstitial as it was for the centered and edge interstitials, but at one of the corners of the initial triangle centered at the original interstitial. This symmetry change of the interstitial may be ascribed to the elastic interaction between these point defects. At smaller distances no further deformation is observed, i.e., the relaxed configurations still contain a vacancy V2 and an interstitial CI'. For $\Lambda / a=1$, the interstitial symmetry change occurs at larger distances, $s \approx 17.7 a$, i.e., for the periodicity cell size $\left(L_{x}, L_{y}\right)=(5,6 \sqrt{3} / 2) 5 a$. This was expected since the interaction between vortices is of longer range.

Finally, we find increasing energies with increasing size $L_{x} \times L_{y}$ of the periodicity cell, which means increasing interaction energy between a vacancy and an interstitial with increasing their distance. We therefore conclude that the interaction between a vacancy and an interstitial is attractive for distances $s$ between 10.6 and 28.3 lattice spacings.

\section{Power-law exponents for defect interactions}

As already mentioned, since we change only the size of the periodicity cell and not its shape, i.e., $L_{x} / L_{y}=0.96$, the total energy of a superlattice of defects should exhibit qualitatively the same distance dependence as the interaction between two single defects in a large periodicity cell. In particular, if the interaction between two point defects at a distance $r$ follows a power law $U \approx r^{-\alpha}$, the energy of a superlattice of such defects will follow the same power law and our method gives the correct exponent $\alpha$.

We find that the defect energies nicely follow power laws $U(s) \propto s^{-\alpha}$, where $s$ is the distance between point defects 


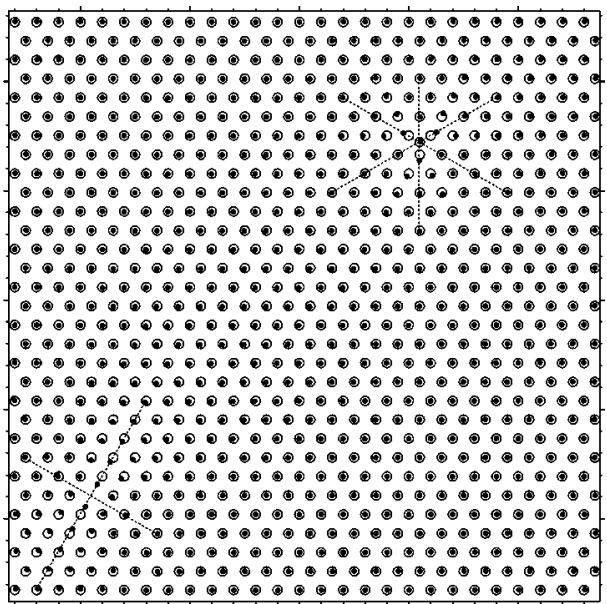

(a)

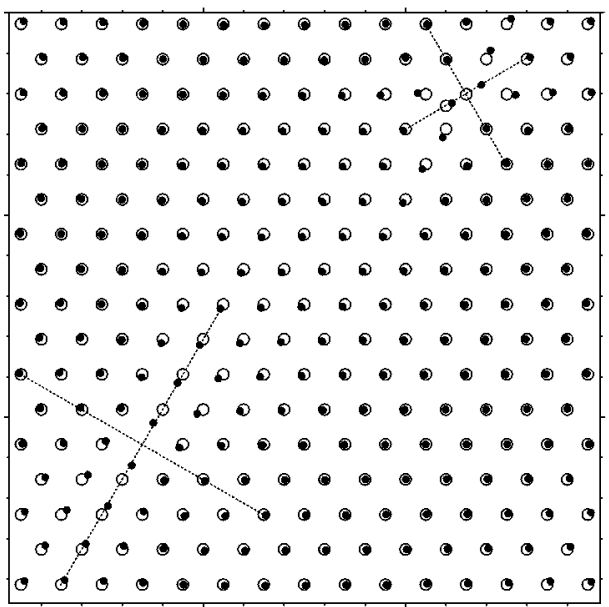

(b)

FIG. 5. Examples of equilibrium vortex configurations obtained with one vacancy-interstitial pair in the periodicity cell and for $\Lambda / a=0.25$. Hollow circles represent the initial configuration with one vacancy-interstitial pair created in the ideal triangular lattice. The final positions obtained after relaxation of the vortices are shown as filled circles. The dotted lines indicate the local mirror planes of the relaxed configuration. We display two configurations corresponding to two different distances $s$ between vacancy and interstitial: (a) $s \approx 21.2 a$, region $27 a \times 27 a$ from the periodicity cell of size $\left(L_{x}, L_{y}\right)=(30,36 \sqrt{3} / 2) a ; \quad$ (b) $s \approx 10.6 a$, region $14.6 a$ $\times 14.6 a$ from the periodicity cell of size $\left(L_{x}, L_{y}\right)=(15,18 \sqrt{3} / 2) a$.

forming a superlattice. This implies that the interaction between two isolated point defects of distance $r$ follows the same power law, i.e., $V(r) \propto r^{-\alpha}$. The results for the exponents $\alpha$ are listed in Table III. These results show $\alpha=2$ for all symmetries of vacancies and interstitials. For the V2, V2', and CI symmetry cases, the exponent differs from 2. One can easily understand these deviations from the value $\alpha=2$ by looking at the final configurations. Indeed, we observe that for the V2, V2', and CI symmetry cases, the deformations of the lattice surrounding the point defect extend to larger distances than in the other cases. In the V2 symmetry case for $\Lambda / a=1$ and for a periodicity cell $\left(L_{x}, L_{y}\right)$ $=(25,30 \sqrt{3} / 2) a$, appreciable deformations of the lattice (compared to the initial triangular lattice) are still encountered at a distance $L_{x} / 2$, while, e.g., for the V3 symmetry no visible deformation of the lattice at distances of $L_{x} / 2$ is ob-
TABLE III. The exponents $\alpha$ of power laws $U=A+B s^{-\alpha}$ which fit the energy of a superlattice of point defects of spacing $s$ by three parameters $A, B$, and $\alpha$. The notations for the defect symmetries are given in the text. The exponents $\alpha$ were obtained from the data in Figs. 2, 3, and 4 and in Tables I and II.

\begin{tabular}{ccc}
\hline \hline Defect symmetries & $\Lambda / a=1$ & $\Lambda / a=0.25$ \\
\hline V6-V6 & 2.0 & 2.0 \\
V3-V3 & 2.0 & 2.0 \\
V2-V2 & 2.3 & 2.3 \\
V2'-V2, & & 2.5 \\
EI-EI & 2.0 & 2.0 \\
CI-CI & 1.8 & 1.8 \\
V2-CI & $3.5<\alpha<4.5$ & $3.5<\alpha<4.5$ \\
\hline \hline
\end{tabular}

served for periodicity cells larger than $\left(L_{x}, L_{y}\right)$ $=(10,12 \sqrt{3} / 2) a$. Since our fitting procedure used the results obtained for periodicity cell sizes from $\left(L_{x}, L_{y}\right)$ $=(15,18 \sqrt{3} / 2) a$ to $\left(L_{x}, L_{y}\right)=(35,42 \sqrt{3} / 2) a$, it includes small cell sizes which are not in the asymptotic regime in the cases of V2, V2', and CI and thus explain the deviations from the exponent $\alpha=2$ obtained in the V2, V2', and CI symmetry cases.

As opposed to the interaction between vacancies or interstitials, we find that for the vacancy-interstitial interaction the exponent $\alpha$ appreciably differs from 2. We computed defect pairs in box sizes up to $\left(L_{x}, L_{y}\right)=(40,48 \sqrt{3} / 2) a$, i.e., up to $t=8$ which gives $40 \times 48=1920$ vortices. The two defects are separated in this case by a distance $s \approx 28.3 a$. With the same arguments developed in the above paragraph, it is straightforward that, since the symmetries involved in the defect pairs are the twofold symmetric vacancy V2 and the threefold symmetric centered interstitial CI, even larger box sizes should be computed in order that all the points involved in our fitting procedure are in the asymptotic regime. The problem is of course to face rapidly increasing computing times. Nevertheless, taking into account six values of pair energies (from $t=3$ up to $t=8$ ) for the fitting procedure, or five values (from $t=4$ up to $t=8$ ), or four values (from $t$ $=5$ up to $t=8$ ), we find slightly oscillating values of the exponent $\alpha$ in the range $3.5<\alpha<4.5$. The values seem to oscillate around $\alpha=4$. It is thus probable that in the asymptotic regime, the exponent of the power law is $\alpha=4$. Such a conjecture calls for further calculations with bigger periodicity cells.

\section{SUMMARY AND CONCLUSIONS}

In conclusion, we computed the arrangement of vortices around various point defects and the corresponding defect energies, in an infinite lattice of short vortices in thin films, using London theory and periodic boundary conditions. We find that vacancies and interstitials of various symmetries define metastable states of the vortex lattice. The same symmetries were found in the case of long parallel Abrikosov vortices. 1,3 We again find that in the thin-film case, the lower the symmetry of the vacancy is, the lower is the corresponding defect energy. As opposed to this, the threefold symmetric centered interstitial (CI) has a lower energy than the twofold symmetric edge interstitial (EI). Furthermore, we find 
that whatever the value of $\Lambda / a$ is, the interaction between two vacancies or two interstitials is attractive for distances between 10 and 35 lattice spacings. These interactions nicely follow a power law $U \approx r^{-\alpha}$ with exponent $\alpha=2$. Considering a vacancy and an interstitial in each periodicity cell, we also find attractive interaction between a vacancy and an interstitial, but the power-law exponent is now larger than two. Our results suggest that in this case the exponent is $\alpha=4$.

The vortex arrangements around point defects in the vortex lattice of thin films in principle may be observed by decoration experiments, ${ }^{17,18}$ and by Lorentz microscopy using an electron microscope. ${ }^{19,20}$ The latter method would even allow us to observe the dynamics and mutual annihilation of vacancies and interstitials. The theory of such reactions, including the generation, motion, and annihilation of edge dislocations in the vortex lattice, may be performed by extending the method presented in this paper.

\section{ACKNOWLEDGMENTS}

This work was supported by the German-Israeli Foundation for Research and Development, Grant No. I-300-101.07/ 93.
*Present address: Laboratoire d'Electrodynamique des Matériaux Avancés, and Laboratoire de Mathématiques et Physique Théorique, Université F. Rabelais, UFR Sciences, Parc de Grandmont, 37200 Tours, France. Electronic address: olive@celfi.phys.univtours.fr

${ }^{1}$ E. Olive and E. H. Brandt, Phys. Rev. B 57, 13861 (1998).

${ }^{2}$ E. H. Brandt, Phys. Status Solidi 35, 1027 (1969); 36, 371 (1969); 36, 381 (1969); 36, 393 (1969).

${ }^{3}$ E. Frey, D. R. Nelson, and D. S. Fisher, Phys. Rev. B 49, 9723 (1994).

${ }^{4}$ M. Slutzky, R. G. Mints, and E. H. Brandt, Phys. Rev. B 65, 453 (1997).

${ }^{5}$ E. H. Brandt, Phys. Rev. B 56, 9071 (1997).

${ }^{6}$ J. Pearl, Appl. Phys. Lett. 5, 65 (1964).

${ }^{7}$ P. G. DeGennes, Superconductivity in Metals and Alloys (Benjamin, New York, 1966), p. 60 ff.

${ }^{8}$ K. B. Efetov, Zh. Éksp. Teor. Fiz. 76, 1781 (1979) [Sov. Phys. JETP 49, 905 (1979)].

${ }^{9}$ S. N. Artemenko and A. N. Kruglov, Phys. Lett. A 143, 485 (1990).

${ }^{10}$ J. R. Clem, Phys. Rev. B 43, 7837 (1991).

${ }^{11}$ A. I. Buzdin and D. Feinberg, J. Phys. (Paris) 51, 1971 (1990).
${ }^{12}$ L. Kramer, Phys. Rev. B 11, 3821 (1971).

${ }^{13}$ Kramer's core interaction should not be confused with the attractive core interaction derived from the nonlocal elasticity of the vortex lattice in Ref. 14, which is important at short vortex distances. This term has the shorter range $\xi / \sqrt{2}$ and for $\kappa=1 / \sqrt{2}$ it exactly compensates the magnetic repulsion.

${ }^{14}$ E. H. Brandt, Phys. Rev. B 34, 6514 (1986).

${ }^{15}$ D. Yu. Irz, V. N. Ryzhov, and E. E. Tareyeva, Phys. Lett. A 207, 374 (1995).

${ }^{16}$ Jung-Chun Wei and Tzong-Jer Yang, Jpn. J. Appl. Phys., Part 1 35, 5696 (1996).

${ }^{17}$ U. Essmann and H. Träuble, Phys. Lett. A 24, 526 (1967); Sci. Am. 224, 75 (1971).

${ }^{18}$ M. Marchevsky, A. Keurentjes, J. Aarts, and P. H. Kes, Phys. Rev. B 57, 6061 (1998).

${ }^{19}$ K. Harada, T. Matsuda, H. Kasai, J. E. Bonevich, T. Yoshida, U. Kawabe, and A. Tonomura, Phys. Rev. Lett. 71, 3371 (1993); K. Harada, O. Kamimura, H. Kasai, T. Matsuda, A. Tonomura, and V. V. Moshchalkov, Science 274, 1167 (1996); A. Tonomura, Physica C 282-287, 339 (1997).

${ }^{20}$ C.-H. Sow, K. Harada, A. Tonomura, G. Crabtree, and D. G. Grier, Phys. Rev. Lett. 80, 2693 (1998). 